\title{
SLC1A5 Silencing Inhibits Esophageal Cancer Growth via Cell Cycle Arrest and Apoptosis
}

\author{
Jieping Lin ${ }^{a}$ Teng Yang ${ }^{b}$ Zheng Peng ${ }^{b}$ Haiyan Xiao ${ }^{c} \quad N^{\prime}$ Jiang $^{b}$ Lifang Zhang ${ }^{b}$ \\ Dickerson $\mathrm{CA}^{\mathrm{c}}$ Ping $\mathrm{Wu}^{\mathrm{b}}$ Qingjun Pan ${ }^{\mathrm{a}}$
}

aKey Laboratory of Prevention and Management of Chronic Kidney Disease of Zhanjiang City, Affiliated Hospital of Guangdong Medical University, Zhanjiang, Guangdong, 'b linical Research Center, Affiliated Hospital of Guangdong Medical University, Zhanjiang, Guangdong, China, 'College of Nursing, Department of Anesthesiology and Perioperative Medicine, Augusta University, Augusta, GA, USA

\section{Key Words}

Slc1a5 • Esophageal cancer • Glutamine $\cdot$ MTORC1

\begin{abstract}
Background/Aims: Solute-linked carrier family A1 member 5 (SLC1A5), which has high affinity to neutral amino acids, is essential for glutamine transport and amino acid metabolism in various cancers. However, the role of SLC1A5 in esophageal cancer has not been reported. Methods: SLC1A5 expression in esophageal cancer tissues was detected by immunohistochemistry and western blotting. The effects of SLC1A5 knockdown on the growth, cell cycle, viability, and glutamine metabolism of esophageal cancer cells were investigated with flow cytometry and western blotting. Furthermore, the consequences of SLC1A5 knockdown on tumor growth and survival were also evaluated in vivo using mice carrying esophageal cancer xenografts. Results: SLC1A5 was expressed in 86.5\% (32/37) of the cancer tissues from esophageal cancer patients. Moreover, SLC1A5 expression in the cancerous tissues was significantly higher than that in the paired adjacent normal tissues. SLC1A5 knockdown with siRNA (PZ siRNA) in TE-1 cells in vitro significantly decreased cell growth and reduced both leucine and glutamine transport, leading to inhibition of mTORC1 signaling. Additionally, siRNA-mediated SLC1A5 knockdown resulted in cell cycle arrest and apoptosis of TE-1 cells. The survival rate of athymic (nu/nu) male nude mice carrying tumors formed from TE-1 cells transfected with SLC1A5 siRNA (PZ siRNA) was also significantly improved compared with mice carrying tumors formed from TE-1 cells transfected with control siRNA. Tumor size/weight was also significantly lower for the former mice group of mice. Conclusion: Our data indicate that SLC1A5 plays an important role in esophageal cancer both in vivo and in vitro. The inhibition of esophageal cancer growth by targeting SLC1A5 could, therefore, be used as a preoperative therapy for esophageal cancer.

Jieping Lin, Teng Yang and Zheng Peng contributed equally to this work.

Prof. Ping Wu

and Dr. Qingjun Pan

Affiliated Hospital of Guangdong Medical University

No.57th, South Renmin Road, Zhanjiang, Guangdong, 524001, (China)

Tel.86-759-2387572, E-Mail wping62@126.com,pqj@gdmu.edu.cn
\end{abstract}




\section{Introduction}

Esophageal cancer is the eighth most common malignancy and the sixth leading cause of cancer-related death in the world [1]. In China, the incidence of esophageal squamous cell carcinoma (ESCC), the most common type of esophageal cancer, exceeds 100 cases per 100, 000 individuals per year. As one of the least studied cancers, treatment options for esophageal cancer are limited, which is a contributing factor to its dismal 5-year survival rate of $18 \%$ [2]. Thus, there is an urgent need to develop novel approaches for the treatment of esophageal cancer.

Glutamine plays an important role in tumor cell metabolism because of its shared characteristics with glucose. Both nutrients help to satisfy two important needs for proliferating tumor cells: bioenergetics (adenosine triphosphate (ATP) production) and the provision of intermediates for macromolecular synthesis [3]. Although glutamine is manufactured and maintained at a high level in normal tissues, under tumorigenic conditions, the consumption rate of glutamine far exceeds cellular manufacturing capabilities. As it is no longer produced at adequate levels endogenously, glutamine changes from a non-essential to an essential amino acid in this environment [3-5].

Solute carrier family 1 member 5 (SLC1A5), also referred to as ASCT2, is a sodium channel which acts as a high-affinity glutamine transporter in tumor cells [6, 7]. Enhanced SLC1A5 expression has been reported in various cancers and mediates biologically significant effects. Fuchs et al [8]., for example, have shown that SLC1A5 is responsible for almost all glutamine uptake in human hepatoma cells and is necessary for SK-Hep cell growth and viability. Furthermore, Kim et al [9]. previously demonstrated that HER2-type breast cancer expresses the highest SLC1A5 levels evaluated to date using immunohistochemistry. SLC1A5 expression is also associated with non-small cell lung cancer [10], lacrimal gland adenoid cystic carcinoma [11], oral squamous cell carcinoma [12], pancreatic ductal carcinoma [13], tongue cancer [14], and colorectal cancer [15]. Notably, while recent research has shown that SLC1A5 expression is increased in esophageal carcinoma [16], the biological significance of SLC1A5 in the development and/or progression of esophageal cancer has not been reported.

In this study, we investigated the role of SLC1A5 in esophageal cancer using a combination of in vitro and in vivo approaches. To our knowledge, this is the first time the biological significance of SLC1A5 has been evaluated in esophageal cancer in this manner. Our results suggest that compounds targeting SLC1A5 may provide novel therapy for esophageal cancer.

\section{Materials and Methods}

\section{Patient specimens}

Tumor specimens were obtained from patients suffering from esophageal cancer who underwent surgical resection at the Affiliated Hospital of Guangdong Medical University. Written informed consent was obtained from all participants. This project was approved by the Ethical Committee of the Affiliated Hospital of Guangdong Medical University. Esophageal cancer and paired adjacent normal tissues were collected during surgery.

\section{Immunohistochemical staining}

SLC1A5 immunohistochemical staining was performed as previously described [13, 17]. Briefly, formalin fixed, paraffin embedded sections ( $2.5 \mathrm{~mm}$-thick) were obtained using a Leica RM2235 microtome (Leica, Germany), transferred to adhesive slides, and dried at $62^{\circ} \mathrm{C}$ for $3 \mathrm{~h}$. To block endogenous peroxidase activity, deparaffinized and rehydrated sections were treated with $0.3 \%$ hydrogen peroxide in methanol for 15 min followed by autoclave sterilization in $0.01 \mathrm{M}$ sodium citrate buffer ( $\mathrm{pH}$ 6.0) for 2 min to expose the antigens. Sections were incubated with a 1:300 dilution of rabbit anti-SLC1A5 antibody (Sigma, USA) overnight at $4^{\circ} \mathrm{C}$ followed by horseradish peroxidase (HRP)-conjugated secondary antibody for $30 \mathrm{~min}$ at room temperature. After incubation with 3, 3'-diaminobenzidine (DAB), SLC1A5 expression was accessed with a light microscope. Negative controls were incubated with phosphate-buffered saline (PBS; Sigma, USA). 


\section{Cellular Physiology Cell Physiol Biochem 2018;48:397-406 \begin{tabular}{l|l|l} 
and Biochemistry $10.1159 / 000491769$ & $\begin{array}{l}\text { DOI } 2018 \text { The Author(s). Published by S. Karger AG, Basel } \\
\text { Puww.karger.com/cpb }\end{array}$
\end{tabular}}

Lin et al.: SLC1A5 Silencing Inhibits Esophageal Cancer Growth

\section{Western blotting}

Total protein was extracted from cell/tissues using RIPA buffer (Roche, IN, USA). Protein concentration was quantified with the BCA protein assay (Beyotime, Shanghai, China). The proteins were then separated by sodium dodecyl sulfate polyacrylamide gel electrophoresis (SDS-PAGE) and transferred to polyvinylidene fluoride (PVDF) membranes. Membranes were blocked in 5\% fat-free milk in Tris-Tween-buffered saline at room temperature for $1 \mathrm{~h}$ followed by incubation with anti-SLC1A5 antibody (diluted 1:300, Sigma, USA), anti-phosphorylated (at threonine 389) ribosomal protein S6 kinase beta 1 (pT389-p70s6K) antibody (diluted 1:1000, Cell Signaling Technology, Inc., USA), anti-phosphorylated (general) p70s6K antibody (diluted 1:1000, Cell Signaling Technology, Inc., USA), or $\beta$-actin antibody (diluted 1:1000, Cell Signaling Technology, Inc., USA) overnight at $4^{\circ} \mathrm{C}$ The membranes were then incubated with peroxidaseconjugated secondary antibodies for $1 \mathrm{~h}$ at room temperature. Immunoreactive bands were detected with a chemiluminescent detection system and then exposed using a Versa DOCTM5000MP System (Bio-Rad Laboratories, Inc. USA). Protein band density was quantified using Image software and normalized to that of $\beta$-actin (loading control).

\section{Cell culture and transfection}

The esophageal cancer cell lines TE-1, Eca109, and EC9706 and the normal esophageal cell line HEEC were purchased from the cell bank of the Chinese Academy of Sciences (Shanghai, China) and were maintained in Roswell Park Memorial Institute (RPMI)-1640 medium (Gibco Life Technologies, USA) containing 10\% heat-inactivated fetal bovine serum (FBS; Gibco Life Technologies, USA) at $37^{\circ} \mathrm{C} 100 \%$ humidity, and $5 \% \mathrm{CO}_{2}$. The cells were seeded into 24-well plates and transfected respectively with 40 nM of each SLC1A5 silencing (si)RNA using Lipofectamine RNAiMAX (Invitrogen, CA, USA) according to the instructions provided by the manufacturer. Based on the known SLC1A5 gene polymorphisms, three SLC1A5 siRNA sequences (1520 siRNA, 1966 siRNA, and PZ siRNA) were designed to knockdown SLC1A5 in cultured esophageal cancer cells. Scrambled nucleotide sequence was used as a nontargeting control. All siRNAs were synthesized by GenePharma Biotechnology (Shanghai, China).

\section{Flow cytometry}

Apoptosis was evaluated using an Annexin V/PI double staining kit (Merck, Germany) according to the manufacturer's instructions. Briefly, single cell suspensions were incubated with Annexin $\mathrm{V}$ solution and stained by propidium iodide (PI) solution for $10 \mathrm{~min}$ at room temperature. Then, the cells were harvested and analyzed with a Becton Dickinson FACS CantoII Flow Cytometer (Beckman Coulter, USA).

To analyze DNA content and cell cycle by flow cytometry, the cells were harvested, washed twice with PBS, and fixed with $70 \%$ ethanol. Immediately before flow cytometric analysis, the cells were washed twice in PBS and then stained for DNA content using $0.5 \mathrm{ml}$ of $400 \mu \mathrm{l} / \mathrm{ml} \mathrm{PI} \mathrm{(Sigma,} \mathrm{USA)} \mathrm{and} 100 \mu \mathrm{g} / \mathrm{ml}$ RNAase A (Sigma, USA) in PBS and 38 mM sodium citrate pH 7.4. Data were again collected on a Becton Dickinson FACS Canto II Flow Cytometer.

\section{Cell culture and L-leucine/L-glutamine uptake assays}

TE-1 cells were cultured in RPMI-1640 medium containing 10\% v/v FBS, penicillin-streptomycin solution, and $1 \mathrm{mM}$ sodium pyruvate and maintained at $37^{\circ} \mathrm{C}$ in $5 \% \mathrm{CO}_{2}\left[{ }^{3} \mathrm{H}\right]$.-L-leucine and $\left[{ }^{3} \mathrm{H}\right]$-L-glutamine uptake assays were performed as described previously [18, 19].

\section{Cell proliferation assay}

At $24,48,72$, and 96 hours after siRNA transfection, the cells were seeded into 96-well plates $(6.0 \mathrm{x}$ $10^{3}$ cells per well) and counted using a Cell-counting Kit-8 assay (Beyotime, China) to assess the viability of transfected TE-1 cells. The absorbance of each well was read on a spectrophotometer (Thermo Fisher Scientific, USA) at $450 \mathrm{~nm}$.

\section{TE-1 xenografts in nude mice}

Athymic (nu/nu) male nude mice (Animal Resource Centre, Southern Medical University, China), 6-8 weeks old, were housed in a specific pathogen-free facility in accordance with the animal ethics committee guidelines of the Guangdong Medical University. Mice were anaesthetized with $2 \%$ isoflurane inhalation and received subcutaneous injections of $1 \times 10^{6}$ TE- 1 cells that were transfected with SLC1A5 siRNA (PZ 
Lin et al.: SLC1A5 Silencing Inhibits Esophageal Cancer Growth

siRNA) or control siRNA resuspended in $100 \mu \mathrm{l}$ of Hanks' balanced salt solution (HBSS) (Gibco, USA). Injections were made into both the right and left ventral flanks of the mice as described previously. After xenograft implantation, survival was monitored for 90 days. In another group of injected mice, tumor growth was analyzed at 3 and 6 weeks after cell implantation in anaesthetized mice. The tumors were harvested at 6 weeks post-implantation, imaged, and weighed prior to western blotting analysis.

\section{Statistical methods}

Data are expressed as the means \pm standard deviation (SD). All experiments were performed with at least three replicates and were analyzed using a Mann-Whitney U-test or one-way ANOVA in GraphPad Prism v. 6. All statistical tests were two-sided. P-values less than 0.05 were considered statistically significant.

\section{Results}

SLC1A5 expression is increased in esophageal cancer

The demographic characteristics of the patients enrolled in this study are shown in Table 1. Immunohistochemical analysis indicates that SLC1A5 was expressed in $86.5 \%(32 / 37)$ of the cancer tissue samples obtained from patients with esophageal cancer (Fig. 1A). Furthermore, our western blotting analysis showed that SLC1A5 expression in the cancer tissues was significantly higher than in the paired adjacent normal tissues (Fig. 1B). These results confirm that there is a significant increase in SLC1A5 expression in esophageal cancer.

\section{SLC1A5 knockdown suppresses} esophageal cancer cell growth

We also examine SLC1A5 protein expression in several human esophageal cancer cell lines, including ECA109, TE-1, EC9706, and HEEC, by western blotting. Our results indicate high expression of SLC1A5 in all four cell lines (Fig. 2A). The TE-1 cell line was selected for further study as it expressed the highest level of SLC1A5 compared to other cell lines examined.

Many studies have shown that SLC1A5 plays an important role in cancer cell
Table 1. Demographic characteristics of patients suffering from esophageal cancer

\begin{tabular}{|c|c|}
\hline Items & Data \\
\hline \multicolumn{2}{|l|}{ Sex } \\
\hline Male & 30 \\
\hline Female & 7 \\
\hline \multicolumn{2}{|l|}{ Age } \\
\hline$<60$ years & 15 \\
\hline$\geq 60$ years & 22 \\
\hline \multicolumn{2}{|l|}{ Lesion location } \\
\hline Upper esophagus & 3 \\
\hline Middle esophagus & 24 \\
\hline Lower esophagus & 10 \\
\hline \multicolumn{2}{|l|}{ Pathological type } \\
\hline Squamous cell carcinoma & 35 \\
\hline Adenocarcinoma & 2 \\
\hline \multicolumn{2}{|c|}{ The degree of tumor differentiation } \\
\hline High-grade & 8 \\
\hline Middle-grade & 19 \\
\hline Low-grade & 10 \\
\hline \multicolumn{2}{|l|}{ TNM staging } \\
\hline Stage I & 4 \\
\hline Stage II & 21 \\
\hline Stage III & 12 \\
\hline
\end{tabular}

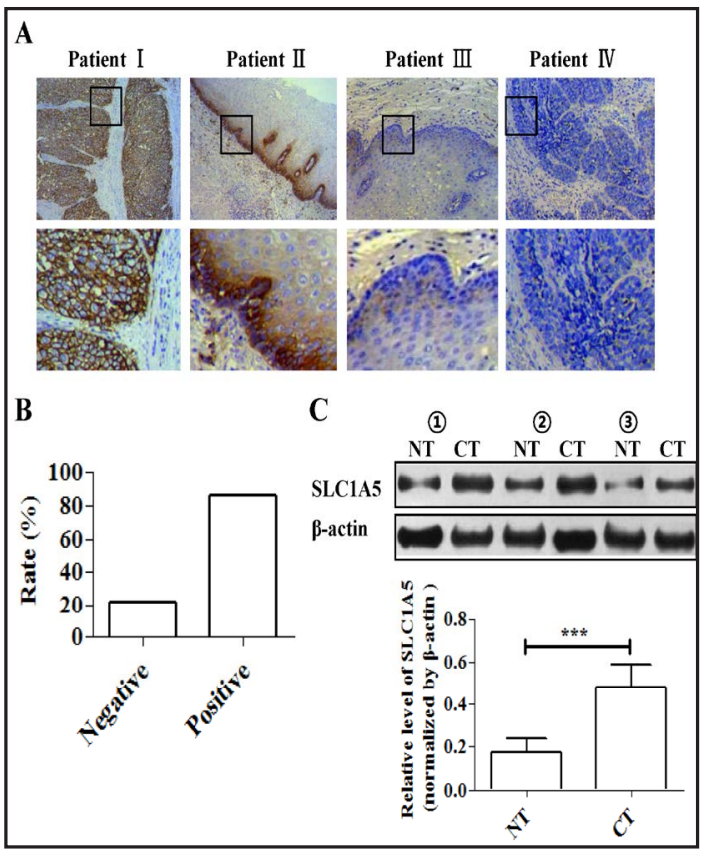

Fig. 1. Detection of SLC1A5 expression in esophageal cancer. (A) Representative images of SLC1A5 immunohistochemically stained esophageal cancer tissues. SLC1A5 positive: Patients I-III; negative: Patient IV. Upper row: magnification $=200 \times$; lower row: enlarged images of the paired local areas shown in the upper row. (B) Statistical analysis of the staining results. (C) Three pairs of SLC1A5 positive esophageal cancer tissues (CT) and paired peritumorous normal tissues (NT) were collected and analyzed for SLC1A5 protein expression by western blot. $^{* * *} \mathrm{p}<0.001$. 
growth [20,21]. Thus, to determine if SLC1A5 directly affects esophageal cancer cell growth in vitro, we utilized three SLC1A5 siRNAs (PZ siRNA, 1966 siRNA, and 1520 siRNA) to silence SLC1A5 expression in TE-1 cells. Our results show that PZ siRNA significantly decreased SLC1A5 expression compared to the control siRNA, while 1520 siRNA and 1966 siRNA did not significantly affect SLC1A5 expression (Fig. 2B).

\section{SLC1A5 knockdown suppresses glutamine metabolism by altering mTOR signaling}

PZ siRNA was selected for our subsequent experiments as it was the most successful at knocking down SLC1A5 expression (Fig. 3A). We also observed TE-1 cell grown to be significantly inhibited by PZ siRNA compared to that of cells treated with control siRNA (Fig. 3B).

Glutamine metabolism plays a very important role in cancer development [22]. To determine the effect of SLC1A5 knockdown on glutamine metabolism, we measured glutamine uptake in TE-1 cells transfected with either PZ siRNA or control siRNA. Our data show that SLC1A5 knockdown significantly decreased glutamine uptake in TE-1 cell (Fig. 3C). Similarly, SLC1A5 knockdown also markedly inhibited leucine uptake (Fig. 3C). As both glutamine and leucine have been reported to activate the mTORC1 pathway [23], we also examined the effects of SLC1A5 knockdown on downstream mTORC1 signaling, focusing on T389 phosphorylation of p70S6K, a downstream target of mTORC1. Our results demonstrate that SLC1A5 knockdown reduces p70S6K phosphorylation in TE-1 cells, suggesting that

Fig. 2. SLC1A5 expression in esophageal cancer cell lines and knockdown efficiency of several SLC1A5 siRNAs. (A) SLC1A5 expression in the esophageal cancer cell lines ECA109, TE-1, EC9706, and HEEC as detected by western blot. (B) SLC1A5 knockdown efficiency in TE-1 cells treated with SLC1A5 siRNA (PZ SiRNA, 1966 siRNA, or 1520 siRNA) or control siRNA as well as untreated (normal) TE-1 cells was detected by western blotting $48 \mathrm{~h}$ post-transfection. The western blotting data was normalized to $\beta$-actin (loading control) for quantitation. Statistical data are presented as the means \pm S.D. of triplicates of each experiment that was repeated 3 times. ${ }^{*} \mathrm{p}<0.05,{ }^{* *} \mathrm{p}<0.01$.

Fig. 3. Inhibition of SLC1A5-mediated glutamine transport in TE-1 cells. (A) SLC1A5 expression in TE-1 cells transfected with control or SLC1A5 siRNA (PZ siRNA) was detected by western blotting $96 \mathrm{~h}$ post-transfection. (B) TE-1 cell growth was analyzed $24,48,72$, and $96 \mathrm{~h}$ after transfected with control or SLC1A5 siRNA (PZ siRNA). (C) Glutamine and leucine uptake by TE-1 cells were examined $96 \mathrm{~h}$ after transfection with control or SLC1A5 siRNA (PZ siRNA). (D) mTORC1 pathway activation (p-p70S6K) was analyzed in TE-1 cells $96 \mathrm{~h}$ after transfection with control or SLC1A5 siRNA (PZ siRNA). Statistical data are presented as the means \pm S.D. of triplicates from an experiment that was repeated 3 times. ${ }^{* * *} \mathrm{p}<0.001$.
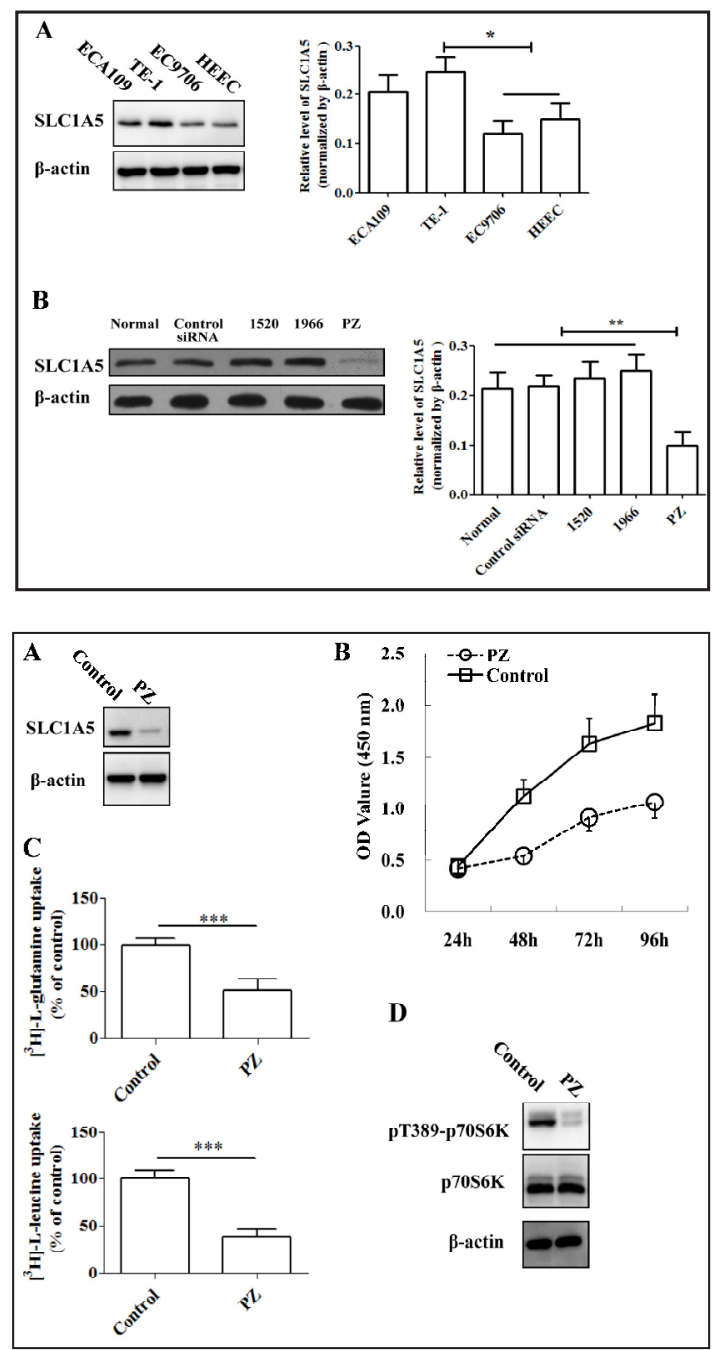
glutamine deprivation inhibits the mTORC1 pathway in esophageal cancer cells (Fig. 3D).

\section{SLC1A5 knockdown induces} cell cycle arrest and apoptosis in esophageal cancer cells

To detect the effects of SLC1A5 knockdown on TE-1 cell proliferation and apoptosis, we evaluated cell cycle distribution after PZ siRNA transfection. After transfection, almost $80 \%$ of the cells transfected with $\mathrm{PZ}$ siRNA were in the G0/G1 phase compared to approximately $50 \%$ of the cells transfected with control siRNA (Fig. 4A). To determine if the G0/G1-arrested cells were und ergoing apoptosis, the cells were assessed using an annexin V-FITC kit. Notably, approximately $40 \%$ of the cells in the PZ siRNA transfected group were annexin $\mathrm{V}$ positive compared to only about 3\% in cells transfected with control siRNA (Fig. 4B). These data suggest that SLC1A5 knockdown induces G0/G1 cell cycle arrest and apoptosis in TE-1 cells.

\section{SLC1A5 knockdown suppresses tumor growth in esophageal cancer xenografts}

We further examined the effects of SLC1A5 inhibition on tumor growth in vivo. For this analysis, TE-1 cells transfected with PZ siRNA or control siRNA were subcutaneously injected into nude mice. Mice were euthanized 3 to 6 weeks after injection depending on the size of the control siRNA tumors. Our results show that the control siRNA tumors were significantly larger than the PZ siRNA tumors (Fig. 5A). Moreover, we also confirmed that the expression of SLC1A5 was indeed downregulated in the PZ siRNA tumors (Fig. 5B). p70S6K phosphorylation was also significantly decreased in the PZ siRNA tumors compared to that in the control siRNA tumors (Fig. 5C). Another group of mice was kept for observation for 90 days to evaluate the survival rate, and the mice transplanted with PZ siRNA-treated TE-1 cells survived significantly longer that those transplanted with control siRNA-treated cells (Fig. 5D).

\section{Discussion}

Glutamine transporters play important roles in metabolism and amino acid homeostasis in a variety of cells and tissues [24]. At a concentration of 0.6-0.9 mM in plasma, glutamine is the most abundant non-essential amino acid under normal conditions [25]. However, glutamine may become conditionally essential in rapidly proliferating cells, such as dividing cancer cells [26,27], because of the rapid uptake and utilization of this amino acid as the cells tries to maintain homeostasis. SLC1A5 is a high-affinity transporter of glutamine that is present in various cancers. Emerging functional studies also indicate that SLC1A5 inhibition retards cancer cell growth and survival [20]. However, while research shows increased SLC1A5 expression in esophageal carcinomas, the biological significance of this expression 
Fig. 5. SLC1A5 is required for tumor growth in vivo. (A) Weights of tumors isolated from mice carrying TE-1 cells transfected with control SiRNA ( $n=10)$ or SLC1A5 siRNA (PZ siRNA) $(n=10)$ at 3 and 6 weeks post-injection. Representative images of the tumors are shown in the lower panel. (B) SLC1A5 expression and quantitation as well as (C) p70S6K phosphorylation in tumors isolated from mice carrying TE-1 cells transfected with control siRNA ( $\mathrm{n}=$ 10) or SLC1A5 siRNA (PZ siRNA) $(n=10)$ at 6 weeks post-injection. (D) The 90 day survival rate of mice carrying TE-1 cells transfected with control siRNA $(\mathrm{n}=12)$ or SLC1A5 siRNA (PZ siRNA) ( $\mathrm{n}=12) .{ }^{* *} \mathrm{p}<0.01,{ }^{* * *} \mathrm{p}<0.001$.

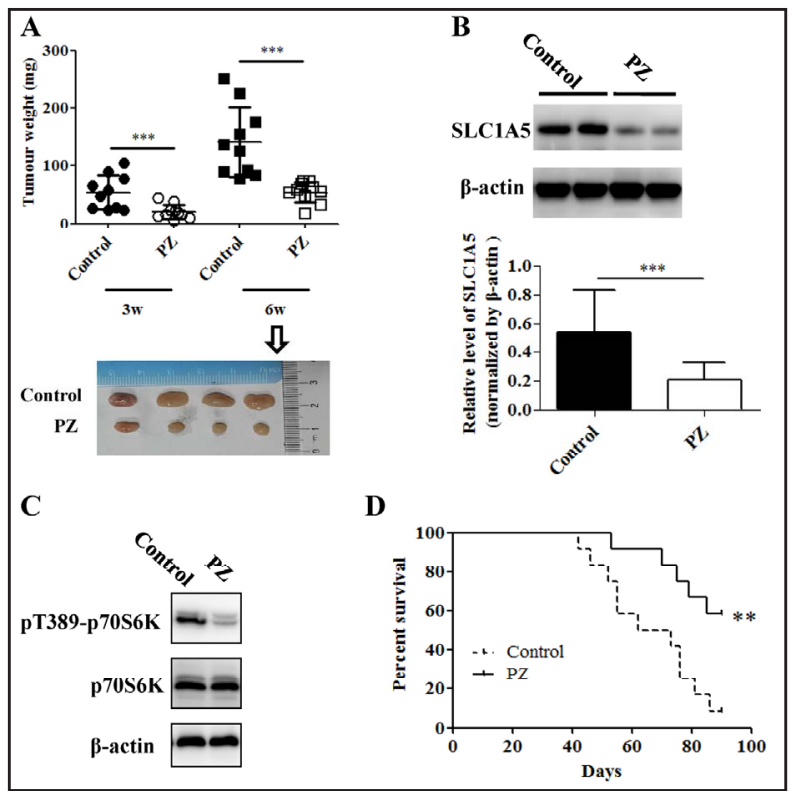

in cancer development has not been determined. In the present study, we report the clinical relevance of SLC1A5 in esophageal cancer using both in vitro and in vivo analyses. To our knowledge, this is the first time the effects of SLC1A5 expression have been evaluated in this type of cancer.

Recent studies have indicated that SLC1A5 is highly expressed in many varieties of cancer. In pancreatic cancer, for example, SLC1A5 was expressed in 54\% (52/97) of tumors, and it is a promising pathological marker for predicting the likelihood of survival [13]. Moreover, Shimizu et al [10]. reported that SLC1A5 is expressed in 66\% of patients suffering from non-small cell lung cancer. Similarly, our data indicate that SLC1A5 was expressed in 86.5\% (32/37) of the esophageal cancer tissues collected in this study. We also detected obvious SLC1A5 expression in the esophageal cancer cell lines ECA109, TE-1, EC9706, and HEEC, thus confirming SLC1A5 expression in esophageal cancer. This expression also indicates a possible function in cancer pathogenesis.

Glutamine transport inhibition has been shown to attenuate the growth of human colon carcinoma [15], non-small cell lung cancer [17], prostate cancer [28], and melanoma cells [19]. To explore the role of the glutamate transporter SLC1A5, we employed a synthetic siRNA system to knockdown SLC1A5 expression in cultured human esophageal cancer cells and found that SLC1A5 downregulation significantly inhibited cell proliferation.

Furthermore, amino acid transporters play an important role in promoting cell cycle progression, particularly through the G0/G1 phase, which is a critical process for protein and DNA synthesis that is required to maintain life. Notably, Miko et al [20]. demonstrated that siRNA-mediated SLC1A5 downregulation in lung cancer cells results in cell cycle arrest in G1 and inhibits mTOR signaling. Similarly, in our analyses, SLC1A5 knockdown resulted in cell cycle arrest in the G0/G1 phase and increased apoptosis in these cells. Thus, SLC1A5 downregulation appears to impair survival and growth in TE-1 cells. This is not altogether surprising as SLC1A5 is an important amino acid transporter and disruption of its expression could be expected to alter related downstream processes. Indeed, Mohamed Hassanin et al [17]. demonstrated that targeting SLC1A5 in non-small cell lung cancer cells induces apoptotic cell death by impairing their ability to uptake sufficient glutamine from the extracellular environment. Induction of apoptosis has also been reported after blocking other amino acid transporters, including xCT (SLC7A11) in triple negative breast cancer as well as SCLC24 and LAT1 (SLC7A5) in human oral epidermoid carcinoma, human osteogenic sarcoma, and C6 rat glioma [17]. Our results also demonstrate that downregulation of SLC1A5 expression with PZ siRNA interferes with the uptake of glutamine, leading to apoptosis.

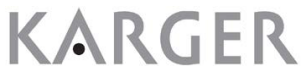


Recent studies have revealed novel functions for glutamine in sustaining cellular growth and modulating the mTOR signaling pathway that are independent of its metabolic role $[10,29]$. In fact, the cytostatic consequences of targeting SLC1A5 activity can be explained, at least in part, by the inactivation of mTOR signaling in addition to the depletion of the intracellular glutamine pool necessary for macromolecule biosynthesis in esophageal cancer. The amino acid is, therefore, multifaceted, being critical for cancer cell growth and proliferation, serving as a signaling molecule, activating mTOR, and acting as an important nitrogen donor for the biosynthesis of amino acids, proteins/macromolecules, and nucleotides (purines/ pyrimidines) [5]. In regards to mTOR signaling, the role of glutamine or glutaminolysis has been shown to be essential for the specific activation of mTORC1 signaling, which regulates protein translation and cell proliferation in many types of cancer. Cancer cells require exogenous glutamine for proliferation, supplying tricarboxylic acid (TCA) cycle intermediates, lipid synthesis, mTOR activity, and neutralization of reactive oxygen species [29]. Furthermore, a study by van Geldermalsen et al [30]. demonstrated that pharmacological inhibition of SLC1A5-mediated transport significantly prevents glutamine uptake, mTORC1 signaling activation, cell growth, and proliferation.

Notably, consistent with our in vitro study, our in vivo results demonstrate that the survival rate of mice exposed to TE-1 cells transfected with SLC1A5 siRNA was significantly improved compared to TE- 1 cells transfected with control siRNA. The tumors isolated from mice injected with SLC1A5 siRNA-transfected cells were also significantly smaller in size and had decreased p70S6K phosphorylation compared to those harboring control siRNAtransfected cells. Thus, it is apparent that silencing SLC1A5 in esophageal tumors could greatly enhance survival.

In summary, we investigated the role of SLC1A5 in esophageal cancer using a combination of in vitro and in vivo approaches. Our results indicate that SLC1A5 expression is increased in tumor samples from esophageal cancer patients, and downregulation of SLC1A5 inhibited cell cycle progression and esophageal cancer growth. To our knowledge, this is the first time the biological significance of SLC1A5 has been evaluated in esophageal cancer in this manner. Our data, in combination with other resent insights in the field, indicate a potential therapeutic benefit of targeting SLC1A5 in esophageal cancer patients.

\section{Acknowledgements}

Source of Funding: Science and Technology Planning Project of Zhanjiang City, China (2014C01025); Natural Science Foundation of Guangdong Province, China (S2013010011568).

\section{Disclosure Statement}

The authors have declared that no competing interest exists.

\section{References}

1 Zhang Y: Epidemiology of esophageal cancer. World J Gastroenterol 2013;19:5598-5606.

2 Dubecz A, Gall I, Solymosi N, Schweigert M, Peters JH, Feith M, Stein HJ: Temporal trends in long-term survival and cure rates in esophageal cancer: a SEER database analysis. J Thorac Oncol 2012;7:443-447.

3 Deberardinis RJ, Sayed N, Ditsworth D, Thompson CB: Brick by brick: metabolism and tumor cell growth. Curr Opin Genet Dev 2008;18:54-61.

4 Medina MA, Sanchez-Jimenez F, Marquez J, Rodriguez Quesada A, Nunez de Castro I: Relevance of glutamine metabolism to tumor cell growth. Mol Cell Biochem 1992;113:1-15. 


\section{Cellular Physiology Cell Physiol Biochem 2018;48:397-406 \begin{tabular}{l|l} 
DOI: 10.1159/000491769 & Ond Biochemistry \\
Published online: July 17, 2018 & $\begin{array}{l}\text { 2018 Tuthor(s). Published by S. Karger AG, Basel } \\
\text { www.karger.com/cpb }\end{array}$
\end{tabular}}

Lin et al.: SLC1A5 Silencing Inhibits Esophageal Cancer Growth

5 Piao L, Fang YH, Parikh K, Ryan JJ, Toth PT, Archer SL: Cardiac glutaminolysis: a maladaptive cancer metabolism pathway in the right ventricle in pulmonary hypertension. J Mol Med (Berl) 2013;91:11851197.

6 Kekuda R, Prasad PD, Fei YJ, Torres-Zamorano V, Sinha S, Yang-Feng TL, Leibach FH, Ganapathy V: Cloning of the sodium-dependent, broad-scope, neutral amino acid transporter Bo from a human placental choriocarcinoma cell line. J Biol Chem 1996;271:18657-18661.

7 Kanai Y, Hediger MA: The glutamate/neutral amino acid transporter family SLC1: molecular, physiological and pharmacological aspects. Pflugers Arch 2004;447:469-479.

-8 Fuchs BC, Perez JC, Suetterlin JE, Chaudhry SB, Bode BP: Inducible antisense RNA targeting amino acid transporter ATB0/ASCT2 elicits apoptosis in human hepatoma cells. Am J Physiol Gastrointest Liver Physiol 2004;286:G467-478.

9 Kim S, Kim DH, Jung WH, Koo JS: Expression of glutamine metabolism-related proteins according to molecular subtype of breast cancer. Endocr Relat Cancer 2013;20:339-348.

10 Shimizu K, Kaira K, Tomizawa Y, Sunaga N, Kawashima O, Oriuchi N, Tominaga H, Nagamori S, Kanai Y, Yamada M, Oyama T, Takeyoshi I: ASC amino-acid transporter 2 (ASCT2) as a novel prognostic marker in non-small cell lung cancer. Br J Cancer 2014;110:2030-2039.

11 Koo JS, Yoon JS: Expression of metabolism-related proteins in lacrimal gland adenoid cystic carcinoma. Am J Clin Pathol 2015;143:584-592.

12 Cetindis M, Biegner T, Munz A, Teriete P, Reinert S, Grimm M: Glutaminolysis and carcinogenesis of oral squamous cell carcinoma. Eur Arch Otorhinolaryngol 2016;273:495-503.

13 Kaira K, Sunose Y, Arakawa K, Sunaga N, Shimizu K, Tominaga H, Oriuchi N, Nagamori S, Kanai Y, Oyama T, Takeyoshi I: Clinicopathological significance of ASC amino acid transporter-2 expression in pancreatic ductal carcinoma. Histopathology 2015;66:234-243.

14 Toyoda M, Kaira K, Ohshima Y, Ishioka NS, Shino M, Sakakura K, Takayasu Y, Takahashi K, Tominaga H, Oriuchi N, Nagamori S, Kanai Y, Oyama T, Chikamatsu K: Prognostic significance of amino-acid transporter expression (LAT1, ASCT2, and xCT) in surgically resected tongue cancer. Br J Cancer 2014;110:2506-2513.

15 Huang F, Zhao Y, Zhao J, Wu S, Jiang Y, Ma H, Zhang T: Upregulated SLC1A5 promotes cell growth and survival in colorectal cancer. Int J Clin Exp Pathol 2014;7:6006-6014.

16 Younes M, Pathak M, Finnie D, Sifers RN, Liu Y, Schwartz MR: Expression of the neutral amino acids transporter ASCT1 in esophageal carcinomas. Anticancer Res 2000;20:3775-3779.

17 Hassanein M, Qian J, Hoeksema MD, Wang J, Jacobovitz M, Ji X, Harris FT, Harris BK, Boyd KL, Chen H, Eisenberg R, Massion PP: Targeting SLC1a5-mediated glutamine dependence in non-small cell lung cancer. Int J Cancer 2015;137:1587-1597.

18 Wang Q, Bailey CG, Ng C, Tiffen J, Thoeng A, Minhas V, Lehman ML, Hendy SC, Buchanan G, Nelson CC, Rasko JE, Holst J: Androgen receptor and nutrient signaling pathways coordinate the demand for increased amino acid transport during prostate cancer progression. Cancer Res 2011;71:7525-7536.

19 Wang Q, Beaumont KA, Otte NJ, Font J, Bailey CG, van Geldermalsen M, Sharp DM, Tiffen JC, Ryan RM, Jormakka M, Haass NK, Rasko JE, Holst J: Targeting glutamine transport to suppress melanoma cell growth. Int J Cancer 2014;135:1060-1071.

20 Hassanein M, Hoeksema MD, Shiota M, Qian J, Harris BK, Chen H, Clark JE, Alborn WE, Eisenberg R, Massion PP: SLC1A5 mediates glutamine transport required for lung cancer cell growth and survival. Clin Cancer Res 2013;19:560-570.

21 Bhutia YD, Babu E, Ramachandran S, Ganapathy V: Amino Acid transporters in cancer and their relevance to "glutamine addiction": novel targets for the design of a new class of anticancer drugs. Cancer Res 2015;75:1782-1788.

22 Choi YK, Park KG: Targeting Glutamine Metabolism for Cancer Treatment. Biomol Ther (Seoul) 2018;26:1928.

23 Jewell JL, Kim YC, Russell RC, Yu FX, Park HW, Plouffe SW, Tagliabracci VS, Guan KL: Metabolism. Differential regulation of mTORC1 by leucine and glutamine. Science 2015;347:194-198.

24 Bode BP: Recent molecular advances in mammalian glutamine transport. J Nutr 2001;131:2475S-2485S; discussion 2486S-2477S.

25 Bergstrom J, Furst P, Noree LO, Vinnars E: Intracellular free amino acid concentration in human muscle tissue. J Appl Physiol 1974;36:693-697. 
26 Fuchs BC, Bode BP: Amino acid transporters ASCT2 and LAT1 in cancer: partners in crime? Semin Cancer Biol 2005;15:254-266.

27 McGivan JD, Bungard CI: The transport of glutamine into mammalian cells. Front Biosci 2007;12:874-882.

28 Wang Q, Hardie RA, Hoy AJ, van Geldermalsen M, Gao D, Fazli L, Sadowski MC, Balaban S, Schreuder M, Nagarajah R, Wong JJ, Metierre C, Pinello N, Otte NJ, Lehman ML, Gleave M, Nelson CC, Bailey CG, Ritchie W, Rasko JE, Holst J: Targeting ASCT2-mediated glutamine uptake blocks prostate cancer growth and tumour development. J Pathol 2015;236:278-289.

29 Moses MA, Neckers L: The GLU that holds cancer together: targeting GLUtamine transporters in breast cancer. Cancer Cell 2015;27:317-319.

-30 van Geldermalsen M, Wang Q Nagarajah R, Marshall AD, Thoeng A, Gao D, Ritchie W, Feng Y, Bailey CG, Deng N, Harvey K, Beith JM, Selinger CI, O’Toole SA, Rasko JE, Holst J: ASCT2/SLC1A5 controls glutamine uptake and tumour growth in triple-negative basal-like breast cancer. Oncogene 2016;35:3201-3208. 